\title{
Cognición y Conciencia
}

\author{
Cognition and Conscience
}

Editor: Jaime Yañez y Adriana Perdomo. Editorial Corporación Universitaria Minuto de Dios - UNIMINUTO. Bogotá 201 1. 27 páginas

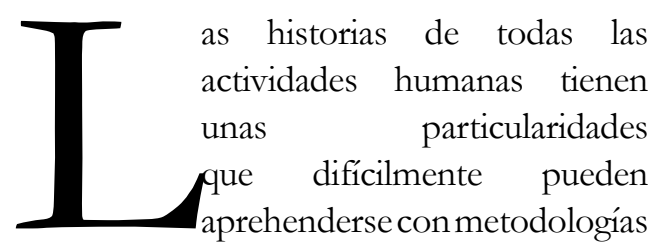

estrictas, con leyes universales y con criterios objetivos de general aceptación. Las historias se asemejan a narraciones donde los protagonistas cambian constantemente, donde los iconos que sostienen las significaciones se presentan en la escena de formas caprichosamente impredecibles, donde los focos de la acción se escurren por tan oscuros resquicios, que parece fueran azarosamente alterados por un travieso espíritu, o un cuerpo extraño, que tuviera como único propósito retar nuestra estereotipada inteligencia.

Los intentos de hacer una historia de las actividades humanas se nutren de una especial capacidad de desmitificación y de una muy intensa sensación de asombro, al igual que de una resignada aceptación de nuevos constreñimientos y nuevos implícitos que determinan los caminos de las nuevas narraciones. Los discursos de prohibición, los puntos ciegos, los discursos retóricos que intentan imponer aldabas invisibles son los enemigos que motivan nuevas cruzadas intelectuales, a pesar de que ellas mismas lleven como equipaje nuevos escudos, igualmente inmovilizadores.

El propósito del libro "Cognicióny Conciencia”, bajo la edición de Jaime Yañez y Adriana Perdomo, no es adelantar una reflexión sobre el operar de la historia, ni ofrecer una narración alejada de cualquier sospecha. Simplemente se ofrece en el escrito una historia particular de la disciplina psicológica y de todas las ciencias cognitivas. Una historia que simplemente se resiste a entronizar determinados paradigmas, criterios únicos de rigurosidad o paquetes particulares de problemas en el variopinto mundo de la ciencia.

La historia de las ciencias cognitivas está salpicada de perspectivas excluyentes, de discursos adjetivados, de señales de caminos prohibidos, de amenazas de miseria intelectual que han evitado que ciertos problemas o teorías fueran incorporados dentro de los discursos oficiales. Pero al igual que en toda actividad humana, los dominios de ciertas perspectivas conceptuales en la Psicología no han durado a perpetuidad, ni han logrado socavar esa inconforme búsqueda del espíritu humano. Muchas de las perspectivas sobre el ser humano que han sido relegadas al margen de la investigación en las ciencias sociales han resurgido periódicamente o han vuelto a ocupar un lugar protagónico, ante el agotamiento que generan las posturas hegemónicas. Obviamente, este resurgir no condena los empeños intelectuales a una historia cíclica, ni a un eterno retorno, solamente evidencia que las estatuas deben ser constantemente refundidas para crear nuevos y más altos monumentos, donde las miradas se posen sobre cada vez más amplios horizontes.

Este libro viene preñado de ese espíritu

1. Profesor Asociado Departamento de Psicología, Universidad Nacional de Colombia. jyanezc@unal.edu.co

2. Coordinadora de investigación Programa de Psicología, Corporación Universitaria Minuto de Dios. aperdomo@uniminuto.edu 
de los pioneros de la Psicología, que ante tan amplio universo ofrendado para la colonización, ofrecieron infinidad de posibilidades, tanto conceptuales como investigativas. Las posturas hermenéuticas convivieron con aquellas que abogaban por convertir la Psicología en un vástago ilegítimo de las ciencias físicas. De la misma manera, las preocupaciones por la mente rompieron lanzas con las perspectivas que nos asemejaban con pequeños mamíferos, los cuales evidencian como prueba de su alta dignidad una enorme capacidad de vivir en laberinticos mundos subterráneos.

Sin pretender contemplar tan rica y colorida historia, podemos referirnos a ciertas problemáticas que motivaron nuestros escritos y nuestro trabajo investigativo. La conciencia y el cuerpo, y las perspectivas conceptuales que posibilitaron la atención de estos fenómenos, son fundamentales en nuestra narración histórica y en nuestra presentación de los caminos más relevantes de la investigación contemporánea. La conciencia y el cuerpo fueron algunos de los problemas que engendraron las primeras teorizaciones de la psicología y que labraron las posibilidades de un fructífero diálogo interdisciplinar. Con la conciencia, la Psicología se preocupó, en sus inicios, por abordar tanto los más variados aspectos de la subjetividad, como las maneras en cómo se podía expresar el espíritu humano ante infinidad de tareas y variadas culturas. Las sensaciones de identidad, el problema de la libertad, los juicios morales, las experiencias de religiosidad y de trascendencia, las debilidades y fortalezas del espíritu humano, el sentido que asegura la existencia humana y la consecución de proyectos de largo alcance, son algunas de las problemáticas derivadas de las primeras comprensiones psicológicas de la conciencia. De igual manera, con la consideración del cuerpo, la Psicología se planteó como tarea el comprender el mundo de las emociones, la particularidad u originalidad del ser humano en el mundo animal, la flexibilidad y armonía de nuestras destrezas no conscientes, la empatía con los otros y aquellas sensaciones de compromiso que nos hacen perseverar en una infinidad de propósitos.

Si bien estas temáticas crearon enormes salas en el panteón siempre abierto de lo humano, no faltaron esos fantasmas, los cuales parecen dominar en las noches los museos. Wundt, Merleau-Ponty, William James, Husserl, Piaget, Koehler, Freud, y muchos otros pioneros de nuestra disciplina, fueron encerrados en la sala donde se colocan las estatuas sin color, sin extremidades o completamente acéfalas. Sus aportes y sus propuestas conceptuales se convirtieron en curiosidades de la historia, que ocasionalmente se señalan como ejemplos de los desafueros de una joven disciplina, la cual lucha afanosamente por asegurar la ciudadanía en el mundo de la ciencia. La consciencia y el cuerpo, con sus preocupaciones por el mito, las experiencias de trascendencia y de búsqueda de sentido, al igual que las reflexiones por la subjetividad, fueron relegadas al mundo del esoterismo o a aquellas disciplinas que tenían por tarea exorcizar esos fantasmas atávicos de nuestro pensamiento salvaje o nuestro patológico operar infantil.

La Antropología y la Psiquiatría fueron esos espacios de exclusión donde los sueños, las pesadillas de dioses jánicos, los compromisos de sacrificio por el semejante, o la indomable y muy humana capacidad de construir historias, encontraron sus posibilidades de existencia. Obviamente, no es nuestro propósito descalificar ni a la Psiquiatría, ni a la Antropología, ni a ninguna otra disciplina social. Hubiéramos podido nombrar disciplinas sociales imaginarias. Simplemente queremos señalar esas estrategias humanas que buscan despojar de responsabilidad los proyectos de realización y que intentan naturalizar las muy humanas maneras de dotar de sentido la existencia. Los mitos, las historias de vida, los proyectos perseverantes de los grandes místicos, los sueños y otras problemáticas donde se evidencia la enorme complejidad del espíritu humano, se relegaron, insistimos en ello, a espacios alterados de una determinada zona cerebral o a vestigios primitivos de una naturaleza infantil, o emocional, sólo consideradas como excepcionalidades o curiosidades no merecedoras 
de atención investigativa.

Pero si los espacios de conocimiento se ven dominados periódicamente por muy variados rituales de exclusión o por profetas de la desventura, que señalan universos de suplicios inundados de aquellos precursores con ropajes poco dignos, igualmente, en estos mismos espacios podemos descubrir esa naturaleza humana que se rebela contra los discursos unificadores y contra los áulicos de la homogenización.

En la investigación contemporánea de la Psicología y de todas las ciencias cognitivas, la historia aparece con su verdadero rostro, con ese rostro que nos recuerda que no solo construimos ídolos, sino que nos preocupamos por adornarlos con los variados trajes del poder. Así como construimos muros para alejarnos del pasado y colocamos símbolos de olvido sobre sus superficies, nuestro espíritu siempre se las ingenia para dejar, como migajas en el camino del conocimiento, esos espejos que reflejan nuestro fracasado intento de encontrar la paz en los encierros.

En las últimas décadas de la investigación cognitiva, la conciencia y el cuerpo han vuelto a ocupar el lugar que no debieron haber perdido. Husserl, Merleau-Ponty, Koehler, James y una infinidad de autores pioneros han sido recuperados para volver a pensar algunos problemas que posibilitaron la constitución de la disciplina psicológica. Obviamente, esta recuperación no consiste en repetir lo que estos autores en su época plantearon. La recuperación de sus ideas se plantea, en las actuales disciplinas que estudian los procesos de conocimiento y de existencia humanas, para pensar en nuevos pilares y originales maneras de abordar el problema psicológico. El sentido de la vida, la sensación de unidad de la experiencia, la constitución bifronte de nuestras identidades, la flexibilidad de nuestra inteligencia, el poder de nuestra intuición, los juicios y las acciones morales, la sabiduría de los años y de nuestro propio cuerpo han sido recuperados para ser pensados con nuevas y más potentes herramientas investigativas.

Este libro parte de esta historia reciente que pretende recuperar los senderos que marcaron las vías de nuestras disciplinas. Bajo este prisma, el libro aborda tanto la historia de la Psicología cognitiva, como la suerte que ha corrido en nuestra disciplina el concepto de conciencia. Igualmente, se aborda la historia del concepto de inconsciente y los diferentes abordajes que se le han dado a las experiencias místicas, y a otros estados especiales de conciencia. En este texto ejemplificamos con una serie de investigaciones los constantes engaños de nuestra conciencia y nuestros perseverantes intentos por dotar de coherencia a nuestra existencia. Los casos de despersonalización, de múltiples personalidades, de negligencias, de olvidos sistemáticos, de sugestión, de sonambulismo y muchos otros nos muestran tanto la fragilidad de nuestra conciencia como su poder restaurador. Los experimentos de la actual ciencia cognitiva, o los casos clínicos, solamente retratan de manera evidente los puntos ciegos de nuestra experiencia y los procesos de confabulación que inundan todas las actividades humanas.

Así, el primer artículo, Anotaciones para una historia del concepto de conciencia en la Psicología, de Jaime Yáñez y Diana Marcela Pérez, intenta hacer una historia rápida y algo provisional sobre el concepto de conciencia, desde las primeras formulaciones de los filósofos griegos hasta las perspectivas actuales en la Ciencia Cognitiva. En este análisis propusimos tres ejes o criterios que permitían identificar las diferentes posturas que han perdurado a través de la historia del término conciencia. Las tres categorías identificadas de manera muy gruesa eran aquellas que han asociado la conciencia con el conocimiento, las que lo han vinculado a procesos de identidad y las que han cuestionado su validez científica o su valor conceptual.

El establecer categorías tan gruesas nos facilitó identificar tendencias estables en la discusión occidental sobre la mente, a la vez que reconocer los ciclos repetitivos de la historia intelectual, alrededor de los problemas centrales de lo que caracteriza lo humano. En esta breve y superficial presentación de la historia del concepto 
de conciencia fuimos mostrando algunos cruces entre posturas y el desarrollo progresivo en la modernidad del problema de la identidad. Es de resaltar en este texto que el avance en la exposición, de las diferentes posturas separadas en el tiempo, se da acompañada de un mayor detalle y profundidad en la argumentación. Si bien en las páginas iniciales se adelantan algunos argumentos dados entre posturas diferentes, éstos solo se hacen claros y evidentes en la presentación de las discusiones más recientes. Las razones de esta estructura expositiva (aparte, claro está de algunas lagunas conceptuales sobre particulares épocas históricas) se deben a que buscábamos conducir al lector hacia un proceso de descubrimiento que nosotros mismos experimentamos. La constante reedición de ciertos argumentos sólo se hace comprensible, cuando el contexto se ha hecho amplio y cuando se ha escuchado lo mismo de muy variadas formas.

El segundo artículo, El inconciente. Historia y posibilidades conceptuales, de Ana Lorena Domínguez y Jaime Yáñez, intenta hacer igualmente una rápida historia del concepto de inconsciente, concentrándonos especialmente en la Psicología y, dentro de esta, en la psicología del procesamiento de la información.

En este artículo se proponen, de la misma manera que en el artículo anterior, unos ejes para el análisis y la organización de los debates y las perspectivas que han ofrecido alguna reflexión sobre los procesos no-concientes del ser humano. Los ejes propuestos son el par positivo-negativo, la oposición pasivo-activo y el tercer eje que gira sobre la comprensión de la subjetividad.

Después de enumerar una serie de propuestas que se centran en algunos de estos ejes en la historia inicial de la psicología, el texto se concentra en exponer las investigaciones en la psicología cognitivo-computacional. A pesar de que los ejes propuestos los consideramos con un alto valor heurístico para organizar las diferentes concepciones sobre el problema, decidimos concentrarnos en uno de estos, debido al mayor nivel de experticia sobre el particular y porque consideramos este empeño un primer escalón de un trabajo de largo aliento.

Dentrodelaexposición sobre elinconsciente en las perspectivas computacionales, abordamos algunas investigaciones sobre los procesos de percepción, la memoria y el pensamiento. Debe quedar claro que las perspectivas abordadas son las que tematizan de manera explícita el problema del inconsciente, y las que lo proponen como un mecanismo fundamental en la explicación del comportamiento humano y en la determinación de la mayoría de sus conductas.

El siguiente artículo, La meditación, correlatos fisiológicos y manifestaciones psicológicas, de Andrés Mauricio Sánchez y Jaime Yáñez, aborda el fenómeno de la meditación y cierto estado especial que alcanzan los maestros o las personas que han dedicado muchos años a esta práctica particular. En la exposición de este fenómeno se señalan algunos detalles de tipo fisiológico e igualmente se adelantan algunas reflexiones sobre las funciones que podría tener la conciencia.

El último artículo, Corriente de conciencia y unidad del sí-mismo: fenomenología de los estados alternados de conciencia, debe leerse como un complemento, o mejor como un documento de contraste con el anterior. En él se presentan casos extraños de conciencia, como los sueños lúcidos, la hipnosis, las experiencias extra-corporales, las experiencias cercanas a la muerte y las experiencias místicas. Aparte de una novedosa caracterización de estos fenómenos, en este artículo se intenta proponer una interpretación fenomenológica de los mismos. Diferenciando los conceptos de ipseidad y de conciencia autobiográfica, el escrito intenta dar cuenta del funcionamiento de la conciencia, en tan particulares estados alterados.

Como podrá notar el lector, los artículos tienen notorias particularidades comolo señalamos anteriormente. Cada uno refleja unos intereses específicos y momentos diferentes del desarrollo de la investigación en torno a la conciencia. Una primera lectura de estos textos posiblemente 
haga pensar, al lector desinformado, que en ellos se presenta una falta de estructura y coherencia en la exposición. Ese tipo de interpretaciones no pueden evitarse, y menos con una aclaración introductoria que muy prontamente habrá de ser olvidada, cuando nuestro acompañante externo se adentre en las primeras líneas. Creemos que el texto que reseñamos se defiende por sí solo. La experiencia que se genera con su lectura es un acto individual de conciencia, que sólo se acompaña de una historia de búsqueda similar a la que los autores han intentado. 